\title{
REUNIONES DE LA ASOCIACIÓN LATINO- AMERICANA DE FACULTADES Y ESCUELAS DE CONTADURÍA (ALAFEC)
}

Dr. Julio Vicente Flores Konja*

\section{INTRODUCCIÓN}

La Universidad Nacional Mayor de San Marcos es miembro de la Unión de Universidades de América Latina (UDUAL), con sede en México, representado por la Universidad Nacional Autónoma de México. Las Asociaciones patrocinadas por la Unión son organismos universitarios y no asociaciones de profesionales, las mismas que forman parte de la UDUAL como entidades académicas de cooperación y estudio, que bajo sus auspicios pueden crear agrupaciones regionales o subregionales para la promoción y desarrollo de una especialidad o rama del saber universitario. Los organismos o asociaciones que reciben el apoyo de la UDUAL son las siguientes:

AFEIDAL. Asociación de Facultades, Escuelas e Institutos de Derecho de América Latina.

AFEIEAL. Asociación de Facultades, Escuelas e Institutos de Economía de América Latina

ALADEFE. Asociación Latinoamericana de Escuelas y Facultades de Enfermeria

ALAFEC. Asociación Latinoamericana de Facultades y Escuelas de Contaduria.
ALAFEM. Asociación Latinoamericana de Facultades y Escuelas de Medicina de América Latina

OFEDO. Organización de Facultades, Escuelas y Departamentos de Odontologia.

RED PUISAL. Red de Programas Universitarios de Investigación en Salud de América Latina.

UDEFAL. Unión de Escuelas y Facultades de Arquitectura de América Latina

\section{ASOCLACIÓN LATINOAMERICANA DE FACULTADES Y ESCUELAS DE CONTADURÍA, ALAFEC}

La Asociación se constituye el día 17 de junio de 1982 en la II Conferencia de Facultades, Escuelas, Departamentos y Programas de Contaduria Pública de América Latina, realizado en la Universidad Autónoma de Santo Domingo, República Dominicana, los dias 13 al 17 de junio de 1982.

En esta Conferencia se resuelve crear una asociación bajo el nombre de ASOCIACIÓN DE FACULTADES Y ESCUELAS DE CONTADURIAA PÜBLICA (Asociación Latino Americana de Facultades de Ciencias Contables), constituyéndose en un organismo

* Profesor Principal y Decano de la Facultad de Ciencias Contables. 
internacional, interuniversitario y regional cuya finalidad principal es proponer a la cooperación y entendimiento de sus asociados, con el objetivo de unificar y elevar el nivel de enseñanza, investigación y capacitación técnica de los CONTADORES y AUDITORES orientado al desarrollo de la América Latina.

\section{ORGANIZACIÓN Y FUNCIONAMIENTO}

Son órganos directivos de la Asociación los siguientes:

LA ASAMBlEA GENERAL. La Asamblea es el máximo organismo de la Asociación y estará integrada por los representantes de las instituciones asociadas en la cual una de ellas tendrá derecho a un voto. La representación será directa y podrá incluir a cualquiera de sus miembros siempre que sea designado por los asociados correspondientes.

EL CONSEJO EJECUTIVO. El Consejo Ejecutivo, cuyo mandato será de dos años, es el órgano delegado por la Asamblea para el Gobierno ordinario de la Asociación. Estará integrado por 4 miembros titulares y 4 suplentes electos por la Asamblea, los cuales representarán funcionalmente a las asociadas de cada una de las siguientes áreas: Región Andina, Región México y El Caribe, Región Centroamericana y Región Cono Sur.

PRESIDENCIA. E1 Consejo Ejecutivo en la primera reunión ordinaria elegirả entre sus miembros aquel que seguirá como Presidente de la ALAFEC durante los dos años de su mandato quien administrará y representará legalmente a la Asociación y simultáneamente presidirá el Consejo Ejecutivo convocando sus reuniones y vigilando las labores de la Secretaria General, con vistas al desarrollo social y económico latinoamericano.

SECRETARÍA GENERAL. La Secretaria General es el órgano permanente a cuyo cargo estará la ejecución efectiva de las resoluciones del Consejo Ejecutivo y la Administración Central de ALAFEC, la cual quedará con Sede permanente en la ciudad de México, ubicándose fisicamente en oficinas destinadas para ejercer sus funciones en el edificio de la Unión de Universidades de América Latina. El cambio de la sede sólo podrá ser resuelto por la Asamblea General mediante el voto aprobatorio de dos tercios de sus miembros presentes.

ORGANISMOS DIRECTIVOS NACIONALES DE LA ASOCLACIÓN. Serán los representantes de la Asociación, en cada país y cumplirán los mismos fines de la Asociación, las Asociaciones Nacionales cuando éstas existan y estén, debidamente constituidas y acreditadas ante la ALAFEC.

\section{OBJETIVOS}

Para cumplir con sus fines la ALAFEC deberá:

a. Colaborar en el desarrollo y progreso de las asociadas, así como estimular su mutuo conocimiento.

b. Servir de centro de consulta y asesoramiento.

c. Servir de órgano coordinador del intercambio y relaciones de facultades y escuelas asociadas tanto entre si como con otros organismos similares. 
d. Proponer las medidas que tengan por objeto coordinar la organización docente, académica y administrativa.

e. Fomentar el intercambio de profesores, alumnos, investigadores, graduados y expositores, así como el de publicaciones, estudios y materiales de investigación y enseñanza.

f. Promover el reconocimiento y respeto de la autonomía universitaria, mediante la vigencia efectiva de las libertades de cátedra e investigación.

g. Auspiciar la adopción de sistemas eficientes para el nombramiento, estabilidad y ascenso de profesores e investigadores.

h. Colaborar para que resulten garantizadas la dignidad humana y la justicia social.

i. Promover la integración cultural y espiritual de la América Latina y su desarrollo económico social a través del esfuerzo coordinado de las asociadas.

j. Estimular y cooperar en la creación de una Facultad Latinoamericana de Contaduria.

\section{ESTRUCTURA DE ALAFEC}

La organización adoptada para el Consejo Ejecutivo establece también la estructura geográfica de ALAFEC. Actualmente la Asociación se encuentra distribuida en las siguientes cuatro regiones geográficas:

ÁREA I. REGIÓN ANDINA: Bolivia, Colombia, Ecuador, Perú, y Venezuela.

ÁREA II. REGIÓN MÉXICO y EL CARIBE: Cuba, México, Puerto Rico y República Dominicana.
ÁREA III. REGIÓN CENTROAMERICANA: Costa Rica, El Salvador, Guatemala, Honduras, Nicaragua y Panamá.

ÁREA IV. REGIÓN CONO SUR: Argentina, Brasil, Chile, Paraguay y Uruguay.

INSTITUCIONES ASOCIADAS. De acuerdo con los archivos de la Asociación y con los registros proporcionados por la Unión de Universidades de América Latina (UDUAL), la ALAFEC agrupa a un total de 204 instituciones de educación profesional. Estas instituciones se distribuyen de la siguiente manera:

ÅREA I: 54 EN LA REGIÓN ANDINA.

ÂREA II: 121 EN LA REGIÓN MÉXICO y EL CARIBE.

ÁREA III: 5 EN LA REGIÓN CENTROAMERICANA.

ÁREA IV: 24 EN LA REGIÓN CONO SUR.

\section{PARTICIPACIÓN DE LA FACULTAD DE CIENCIAS CONTABLES DE LA UNMSM}

$\mathrm{Al}$ inicio del año 2002 la facultad recibió una invitación en la que se señala a nuestra universidad como la de mayor prestigio del Perú para participar activamente en la Asociación. La participación la cual fue inmediatamente autorizada por el Rectorado, considerando que era necesario mantener vínculos con todas las universidades a fin de actualizar las diversas áreas del conocimiento e intercambiar experiencias en los avances de la educación superior, la investigación, las curriculas de estudio, intercambios de docentes y otros aspectos, cuya responsabilidad rectora tradicionalmente ha correspondido a San Marcos. 
REUNIÓN EN MÉXICO DEL CONSEJO EJECUTIVO AMPLIADO DE LA ALAFEC, DEL 18 AL 19 DE ABRIL DE 2002, UNIVERSIDAD NACIONAL AUTÓNOMA DE MÉXICO-UNAM

Se efectuó siendo sede la Facultad de Contaduria y Administración de la Universidad Nacional Autónoma de México, asistiendo representantes de Universidades de 12 paises (Anexo 01).

El CPC y maestro Arturo Díaz Alonso, en su calidad de Presidente de la Asociación, dio la bienvenida a los integrantes del Consejo Ejecutivo Ampliado, e invitados de la UDUAL, de la Asociación Nacional de Facultades y Escuelas de Contaduría y Administración (ANFECA) y a los de la Facultad de Contaduria y Administración de la UNAM.

Declarado el acto inaugural, el maestro Jorge Peralta, Secretario Académico de la UDUAL en representación del Secretario General, Dr. Juan José Sánchez, expresó su beneplảcito de la ALAFEC, reafírmando el apoyo que la UDUAL puede brindar a los organismos de cooperación y estudio como la ALAFEC.

Los participantes realizaron su presentación planteando diversos aspectos académicos que tienen importancia para la carrera de Contaduria en los diferentes paises representados.

\section{ACUERDOS}

1. La Presidencia de la Asociación presentó la propuesta del Plan de Trabajo ALAFEC 2002-2003, acordándose su aprobación.

2. Respecto al tema central de la VIII Conferencia de ALAFEC, se acordó:
2.1. Crear la Comisión de trabajo académico para la realización y exposición del tema central, la cual está integrada de la forma siguiente:

L.C. José Lino Rodriguez Sánchez, Coordinador General.

M.G. Fernando Rodríguez Martínez, Coordinador de la Región I.

Mtra. Emma Polanco, Coordinadora de la Región II.

Mtro. Rodimiro Mejía, Coordinador de la Región III.

Mtra. Gladis Soto, Coordinadora de la Región IV.

Dr. Antonio Rodríguez Rojas. Integrante de la Comisión.

2.2. Que se incluyera como parte importante dentro de la justificación del tema central un apartado sobre la Convalidación de planes y programas de estudios.

2.3. La Comisión de trabajo académico deberá presentar para la próxima reunión, una propuesta totalmente terminada sobre la metodologia para la elaboración y desarrollo del tema central. Esta debe incluir entre otros aspectos: $\mathrm{El}$ título final del tema central, los objetivos, los mecanismos de participación de todos los asociados a ALAFEC, programa de actividades, etc.

3. Respecto a la próxima Asamblea General de ALAFEC, se tomaron los acuerdos siguientes:
3.1.La Asamblea se realizará conjuntamente con la VIII Conferencia de ALAFEC en el mes de septiembre de 2003. 
3.2. Crear la Comisión para la reforma estatutaria la cual se encargará de realizar el estudio y propuesta de reforma de los estatutos de ALAFEC, esta misma se integrará por el Secretario General de la Asociación y los Vicepresidentes de cada una de las regiones:

Mtro. Tito Livio de la Torre Hidalgo, Coordinador General.

Dr. Benjamín López Arciniegas, Región I.

Mtra. Emma Polanco, Región II.

Mtro. Gustavo Ciles, Región III.

C.P. Carlos Anibal Degrossi, Región IV.

\subsection{El L.C. Leonel Sebastián} Chavarría se encargará de promover la Asamblea General y VIII Conferencia de ALAFEC a las instituciones educativas de Contaduría de América Latina, y de convocar su asistencia.

4. El Mtro. Arturo Diaz Alonso coordinará la Comisión de trabajo encargada de crear la Red Internacional de Investigadores de nuestras disciplinas en América Latina. A esta comisión se integra el Dr. Julio Flores Konja.

5. El Dr. Tomás Sánchez será el coordinador de la Comisión para el diseño y elaboración de la Carta Latinoamericana de Facultades y Escuelas de Contaduria Pública, quien además se responsabilizará de diseñar una propuesta de imagen y logotipo de la Asociación. A esta comisión se integra el Mtro. Macario Ortega Mariño.

6. El Dr. Jorge Ayala será el responsable de recavar y analizar la información referente a la Acreditación de Instituciones de Educación Superior de Contaduría en América Latina.

7. Se elaborará la página web de la Asociación, la cual tendrá como dirección electrónica la siguiente: www.alafec.unam.mx. Los contenidos sugeridos para la misma serán los siguientes:

a) Información sobre la Asociación (Objetivos, Historia, Estructura, etc.)

b) Los Estatutos.

c) Directorio.

d) Actas del Consejo.

e) Planes de estudio de las instituciones afiliadas.

La institución responsable de administrar la Página Web será la Facultad de Contaduría y Administración de la Universidad Nacional Autónoma de México.

8. Se acordó el Programa de Actividades siguiente:

1. Reunión del Consejo Ejecutivo Ampliado. Santiago de Chile Fecha: Del 16 al 20 de Octubre de 2002.

Anfitrión responsable: Mtra. Gladis Soto Villarroel.

Orden del dia: Avances de las labores del Consejo Ejecutivo Ampliado y de las Comisiones.

2. Reunión del Consejo Ejecutivo Ampliado. La Habana

Fecha: Del 9 a 11 de abril de 2003. 
Anfitrión responsable: Mtra. Mirna Ricard Delgado.

Orden del día: Por definir.

3. Asamblea General y VIII Conferencia. México

Fecha: Setiembre de 2003.

REUNIÓN EN CHILE DEL CONSEJO EJECUTIVO DE LA ALAFEC DEL 17 AL 18 DE OCTUBRE DE 2002 , UNIVERSIDAD NACIONAL DE SANTIAGO DE CHILE

Esta reunión se llevó a cabo en la sede de la Universidad de Santiago de Chile-Facultad de Contabilidad y Auditoria, asistiendo los representantes de Argentina, Bolivia, Colombia, Cuba, Ecuador, Honduras, México, Nicaragua, Paraguay, Perú, República Dominicana, Venezuela y Chile. (Anexo 01).

El Decano de la Facultad de Administración y Economia de la Universidad de Santiago de Chile, Sr. Jorge Pérez Barberito, destacó el valor de la ética, la eficacia y la eficiencia en la ciencia contable al inaugurar el evento. La profesora Gladis Soto, presidenta de la Comisión Organizadora, destacó que la convocatoria fue todo un éxito dada la alta responsabilidad de las autoridades universitarias contables de 13 países, lo que privilegia la profesión y la formación de los Contadores Públicos.

Alonso, subrayó la necesidad de en beneficio de la
Cumpliendo los acuerdos del mes de abril en México, la Comisión presentó el Plan de Trabajo ALAFEC 2002-2003.

En el desarrollo de la reunión tomaron se tomaron diversos acuerdos.

1. El Presidente de la ALAFEC puso a consideración el Acta de la reunión del Consejo Ejecutivo de la Asociación Latinoamericana de Facultades y Escuelas de Contaduria, celebrada el 18 y 19 de abril de 2002, teniendo como sede la Facultad de Contaduria y Administración de la UNAM.

Acuerdo único:

El Acta fue aprobada por unanimidad.

2. El Lic. José lino Rodríguez Sánchez, la Mtra. Emma Polanco, el M.G. Fernando Rodriguez Martínez y la Mtra. Gladis Soto Villarroel, presentaron la propuesta metodológica para estructurar la PONENCIA CENTRAL de ALAFEC 2003, "Creación de un Plan de Estudios Latinoamericano para la Formación del Contador Público", la misma que se complementó con la propuesta de Reglamento General para los Coloquios ALAFEC.

Acuerdos:

2.1. La «Propuesta de Estructura de la Ponencia Central. ALAFEC 2003" y la propuesta de "Reglamento Gener para los Coloquios aprodan. neral. Asumsmo cumento antes me 
Anfitrión responsable: Mtra. Mirna Ricard Delgado.

Orden del día: Por definir.

3. Asamblea General y VIII Conferencia. México

Fecha: Setiembre de 2003.

\section{REUNIÓN EN CHILE DEL CONSEJO EJECUTIVO DE LA ALAFEC DEL 17 AL 18 DE OCTUBRE DE 2002 , UNIVERSIDAD NACIONAL DE SANTIAGO DE CHILE}

Esta reunión se llevó a cabo en la sede de la Universidad de Santiago de Chile-Facultad de Contabilidad y Auditoria, asistiendo los representantes de Argentina, Bolivia, Colombia, Cuba, Ecuador, Honduras, México, Nicaragua, Paraguay, Perú, Repüblica Dominicana, Venezuela y Chile. (Anexo 01).

El Decano de la Facultad de Administración y Economía de la Universidad de Santiago de Chile, Sr. Jorge Pérez Barberito, destacó el valor de la ética, la eficacia y la eficiencia en la ciencia contable al inaugurar el evento. La profesora Gladis Soto, presidenta de la Comisión Organizadora, destacó que la convocatoria fue todo un éxito dada la alta responsabilidad de las autoridades universitarias contables de 13 paises, lo que privilegia la profesión y la formación de los Contadores Públicos.

En esta reunión inaugural el presidente de la ALAFEC, Arturo Diaz Alonso, subrayó la necesidad de aunar esfuerzos en beneficio de la profesión y de la formación de los futuros profesionales de la contabilidad y la administración.
Cumpliendo los acuerdos del mes de abril en México, la Comisión presentó el Plan de Trabajo ALAFEC 2002-2003.

En el desarrollo de la reunión tomaron se tomaron diversos acuerdos.

1. El Presidente de la ALAFEC puso a consideración el Acta de la reunión del Consejo Ejecutivo de la Asociación Latinoamericana de Facultades y Escuelas de Contaduria, celebrada el 18 y 19 de abril de 2002, teniendo como sede la Facultad de Contaduría y Administración de la UNAM.

Acuerdo único:

El Acta fue aprobada por unanimidad.

2. El Lic. José Lino Rodríguez Sánchez, la Mtra. Emma Polanco, el M.G. Fernando Rodriguez Martínez y la Mtra. Gladis Soto Villarroel, presentaron la propuesta metodológica para estructurar la PONENCIA CENTRAL de ALAFEC 2003, "Creación de un Plan de Estudios Latinoamericano para la Formación del Contador Público", la misma que se complementó con la propuesta de Reglamento General para los Coloquios ALAFEC.

Acuerdos:

2.1.La "Propuesta de Estructura de la Ponencia Central. ALAFEC 2003" y la propuesta de "Reglamento General para los Coloquios ALAFEC" fueron aprobadas en lo general. Asimismo, a cada documento antes mencionado se realizaron algunas modificaciones en lo particular, mismas que se adecuarán a 
las versiones finales de los documentos. El coordinador de la comisión remitirá dichos documentos a cada uno de los integrantes del Consejo para su consecuente uso y aplicación.

2.2. Los Vicepresidentes y Representantes Nacionales coordinarán la realización de los coloquios nacionales y regionales de acuerdo con el siguiente calendario:

a) Los coloquios nacionales, a más tardar en abril de 2003.

b) Los coloquios regionales, a más tardar en junio de 2003.

Nota: En el Anexo 02, información adicional sobre la Ponencia Central.

3. El Mtro. Arturo Díaz Alonso, presentó el proyecto para la creación de la Red Internacional de Investigadores de ALAFEC.

Se tomaron 4 acuerdos:

3.1. El proyecto fue aprobado en lo general.

3.2. Se especificaron las siguientes Áreas de investigación:

ÁREAS BÁSICAS:

- Auditoría

- Contabilidad

- Control

- Costos

- Contribuciones (Tributación)

- Finanzas

ÁREAS COMPLEMENTARIAS:

- Ética

- Gestión ambiental.

- Formación profesional.

3.3. Se creó una Comisión Coordinadora que está integrada por las siguientes instituciones:
- Facultad de Contaduria y Administración de la Universidad Nacional Autónoma de México.

- Facultad de Ciencias Económicas y Sociales de la Universidad de Santo Domingo

- Facultad de Contabilidad y Finanzas de la Universidad de la Habana.

- Facultad de Ciencias Económicas de la Universidad de Buenos Aires.

3.4. Se iniciará la creación de Directorios Nacionales de Investigadores con el fin de crear el directorio de Investigadores ALAFEC. Los Representantes Nacionales serán los responsables de desarrollar cada directorio en el país que le corresponda.

Nota: En el Anexo 03, información adicional.

4. El Mtro. Tito Livio de la Torre presentó los avances de la propuesta para realizar la reforma estatutaria de ALAFEC, con el fin de adecuar la normatividad existente a la realidad latinoamericana vigente. Esta propuesta fue enriquecida con los comentarios y sugerencias de los asistentes.

Acuerdo único:

El Mtro. Tito Livio de la Torre recogerá los comentarios efectuados en la reunión y los incluirá en una nueva propuesta que presentará en la próxima reunión del Consejo Ejecutivo de ALAFEC. Cabe destacar que antes de dicha presentación, el Mtro. Tito Livio enviará la propuesta de estatu- 
tos a cada miembro del Consejo para su lectura previa.

El L.C. Leonel Sebastián Chavarria presentó el "Proyecto para el Posicionamiento, Afiliación y Consolidación de la ALAFEC" el cual tiene por objetivos: 1) Posicionar la imagen de ALAFEC en Latinoamérica, 2) Afiliar al mayor número posible de facultades y escuelas de contaduria en Latinoamérica, y 3) Lograr que la membresía afiliada asista a la próxima Asamblea General de ALAFEC.

Acuerdos:

1. El proyecto fue aprobado.

2. Los Representantes Nacionales serán los responsables de difundir la Gaceta ALAFEC y realizar una campaña de afiliación en cada uno de sus paises. Se estableció como compromiso que cada uno de los representantes afiliará a un mínimo de 5 instituciones dentro del periodo comprendido entre la fecha de esta reunión y la próxima reunión del Consejo a celebrarse en abril del año 2003.

3. Los Vicepresidentes adoptan el compromiso de nombrar, en los países donde haga falta, al Representante Nacional correspondiente. Asimismo, los Vicepresidentes asesorarán a los nuevos Representantes Nacionales para que realicen la campaña de afiliación en estos países.

4. La Presidencia de ALAFEC, a través de la Secretaría Ejecutiva y de la Coordinación de Relaciones Internacionales, impulsará la campaña de afiliación por medio de una estrategia dirigida a los Rectores de las Universidades que formen parte de la Unión de Universidades de América Latina con el fin de que apoyen a los Directores y Decanos a formar parte de esta Asociación.

5. El objetivo a lograr es que se afilie a un minimo de 200 escuelas o facultades en América Latina.

Nota: En el Anexo 04, información adicional.

6. El L.C. Leonel Sebastián Chavarría presentó avances sobre la Página Web de ALAFEC, la Gaceta ALAFEC y propuestas de logotipos de la Asociación.

Acuerdos:

6.1. Los integrantes del Consejo Ejecutivo revisarán la página web presentada y remitirán sus comentarios $\mathrm{y}$ sugerencias al responsable.

6.2. El L.C Leonel Sebastián adecuará la información de la gaceta ALAFEC, incluyendo los acuerdos tomados en esta reunión, y remitirá el archivo electrónico que contenga dicha gaceta a cada uno de los miembros del Consejo, con el fin de que pueda ser difundida en sus respectivos paises $\mathrm{y}$ regiones.

6.3. El L.C Leonel Sebastián se encargará de coordinar el diseño final del logotipo de la Asociación con base en las sugerencias que realizaron los miembros del Consejo. 
Dicho logotipo será enviado a los integrantes del mismo.

7. La Lic. Gladys Soto Villarroel presentó un documento encaminado a fortalecer la formación académica del contador público a nivel universitario y a promover el intercambio académico de docentes y estudiantes de las facultades y escuelas integrantes de ALAFEC. El ideario de dicho documento se incorporará a la Carta Latinoamericana de Facultades y Escuelas de Contaduria.

8. El centro de alumnos de contador público y auditor, de la Universidad de Santiago de Chile presentó una solicitud ante el Consejo Ejecutivo Ampliado para que se considere la realización del "Primer Congreso Latinoamericano de Estudiantes de Contador Público". La propuesta fue recibida con beneplácito $y$ será tratada en la próxima reunión del Consejo Ejecutivo de ALAFEC.

9. En el marco de esta reunión y como acto protocolario, el C.P. y Maestro Arturo Diaz Alonso en su calidad de Director General de la Asociación Nacional de Facultades y Escuelas de Contaduria y Administración (ANFECA) y el Maestro Fernando Rodríguez Martínez en su calidad de Presidente de la Asociación de Facultades y Escuelas de Contaduria de Colombia (ASFACOP), firmaron un convenio de colaboración académica entre ambas Asociaciones.

10.Los asistentes a la reunión externaron un merecido recono- cimiento y agradecimiento a la labor realizada por los Maestros. Jorge Pérez Barbeito y Gladis Soto Villarroel quienes fungieron como anfitriones de esta reunión.

\section{ACUERDOS FINALES}

11. El L.C. Lino Rodríguez Sánchez, establecerá comunicación con los miembros del Consejo que estuvieron ausentes en esta reunión a fin de informarles sobre el desarrollo de la misma y acuerdos tomados. Así mismo les solicitará, según sea el caso, el avance de sus trabajos.

Se acordó el siguiente Calendario de Reuniones:

11.1. Reunión del Consejo Ejecutivo Ampliado.

Fecha: Del 9 al 11 de abril de 2003.

Lugar: La Habana.

Anfitrión responsable: Mtra. Mirna Richard Delgado.

Orden del día: Por definir.

11.2. Asamblea General y VIII Conferencia.

Fecha: Septiembre de 2003.

Lugar: Mérida Yucatán, México.

11.3. Reunión del Consejo Ejecutivo Ampliado.

Fecha: Marzo del 2004.

Lugar: Buenos Aires.

Anfitrión responsable: C.P. Carlos Anibal Degrossi.

Orden del dia: Por definir. 
C.P.C. y Mtro. Arturo Diaz Alonso.

Presidente de la Asociación.

Mtro. Tito Livio de la Torre Hidalgo Secretario General de la Asociación.

Dr. Benjamín López Arciniegas Vicepresidente del Área I. Región Anduna

Mtra. Emma Polanco

Vicepresidenta del Area II. Region Mexico y IEl Caribe

Dr. Gustavo Ciles

Vicepresidente del Área III. Región Centroamericana

Dr. Tomás Sánchez Torres

Representante Nacional en Boliva

Dr. Carlos Anibal Degrossi

Vicepresidente del Area

III. Region

Centroamérica

Lic. Fernando A. Rodriguez Martínez

Representante Nacional en Colombia

Dr, Julio Flores Konja

Representante Nacional en Peru

I C.P. Rey Martínez Mendoza

Representante Nacional en México

Lic. Mirna Ricard Delgado

Representante Nacional en Cuba

Dr. Jorge Ayala Cruz

Representante Nacional en Puerto Rico

Mtro. Rodimiro Mejía Merino

Representante Nacional en Honduras

Dr. Carlos Araya Villalobos

Representante Nacional en Chile

Dr. Jorge Pérez Barbeito

Representante Nacional en Chile

Dr. Antonio Rodriguez Rojas

Representante Nacional en Paraguay

L.C. José Lino Rodriguez

Secretario Ejecutivo

L. C. Leonel Sjébastián Clıavarna Coordinador de Rolaciones Internacionaies

Dr. Víctor Rago

Representante Nacional en Venezuela

Dr. José Villavicencio Rosero

Representante Nacional en Ecuador
Director de la Facultad de Contaduria y Administración de la Universidad Nacional Autónoma de México.

Director de la División Econōmico-administrativa de la Universidad Popular Autónoma del Estado de Puebla.

Decano de la Facultad de Contaduria Pública de la Universidad Central de Bogota.

Decana de la Facultad de Ciencias Económicas y Sociales de la Universidad Autónoma de Santo Domingo

Decano de la Facultad de Ciencias Económicas de la Universidad Nacional Autónoma de Nicaragua.

Decano de la Facultad de Ciencias Económicas y Flnancieras de la Universidad Mayor de San Andres.

Decano de la Facultad de Ciencias Económicas de la Universidad de Buenos Aires.

Decano de la Facultad de Contaduria Pública de la Universidad Santo Tomás.

Decano de la Facultad de Ciencias Contables de la Universidad Nacional Mayor de San Marcos.

Decano de la Facultad de Contaduria y Administración de la Universidad Autónoma del Estado tración de la
de Morelos.

Decana de la Facultad de Contabilidad y Finanzas de la Universidad de la Habana.

$\mathbf{M}$

M

M

$\mathrm{CH}$

$\mathbf{M}$

$\mathrm{CH}$

$\mathbf{M}$

Aus.

Aus.

Decano de la Facultad de Administración de Empresas de la Universidad de Puerto Rico en San Juan, Recinto de Río Piedras.

Decano de la Facultad de Ciencias Económicas y Administración de la Universidad Nacional de Honduras.

Decano de la Facultad de Administración y Economía de la Universidad de Santiago de Chile.

Decano de la Facultad de Administración y Economía de la Universidad de Santiago de Chile.

Decano de la Facultad de Ciencias Económicas de la Universidad Nacional de Asunción.

Secretario de Intercambio Académico. Facultad de Contaduria y Administración Universidad Nacional Autónoma de México y Secretario General Asociación Nacional de Facultades y Escuelas de Contaduría y Administración.

Conrrlinador General de Intercambio Académico. Facul ad de Contaduria y Administración. Universidad Nacional tónoma de Mérico

Decano de la Facultad de Ciencias Económicas. Universidad Central de Venezuela.

Decano de la Facultad de Ciencias Administrativas. Universidad Central de Ecuador.
$\mathrm{M} \quad \mathrm{CH}$

$\mathbf{M}$

$\mathrm{CH}$

$\mathrm{CH}$

M

Aus.

M

Aus.

M

Reem-

plazado

Asistió $\mathrm{CH}$

Anterior Nuevo

Decano Decano

M

$\mathrm{CH}$

M

$\mathrm{CH}$

M

$\mathrm{CH}$

Aus

$\mathrm{CH}$

$\mathrm{CH}$
Aus. $\mathrm{CH}$ 


\section{'LA CREACIÓN DE UN PLAN DE ESTUdIOS LATINOAMERICANO PARA LA FORMACIÓN DEL CONTADOR PUBLICO"}

Por acuerdo del Consejo Ejecutivo, en la reunión efectuada en México, se acordó celebrar la ASAMBLEA GENERAL en el mes de SETIEMBRE de 2003 teniendo como tema central "La creación de un Plan de Estudios Latinoamericano para la formación académica del Contador Público”

\section{ANTECEDENTES}

Las Conferencias Latinoamericanas de ALAFEC se definen como reuniones de académicos, investigadores y directivos de las Escuelas y Facultades de Contaduria de América Latina. En las mismas se abordan temas aspectos de alto interés para los participantes, profesionales del área y afines, así como para quienes concurren como invitados o en calidad de representantes de centros de formación especializada en la región.

En cumplimiento del mandato otorgado en la reunión del Consejo Ejecutivo, efectuada en México en marzo del presente año, presentamos la propuesta de estructura para la ponencia central ALAFEC 2003, habiendo determinado abordar el tema de *La creación de un Plan de Estudios Latinoamericano para la formación del Contador Público", principalmente por las tendencias recientes observadas en los procesos globalizadores que marcan preferencias por un profesional, portador de condiciones similares para un ejercicio competitivo en el mercado de trabajo.

\section{JUSTIFICACIÓN}

La educación en general y la enseñanza de la contaduria en particular, han resentido los cambios en la estructura política y económica mundial. Todas las Instituciones de Educación Superior desean satisfacer sus requerimientos en materia educativa, por lo que observamos cotidianamente nuevos planteamientos orientados a responder estas nuevas exigencias.

Los recientes cambios en la estructura económica mundial han dado como resultado modificaciones en el terreno de la ciencia, la tecnología y, por supuesto la cultura y la educación, comprometiendo a las instituciones de educación superior a revisar sus curricula, a organizar eventos de intercambio de información con otras instituciones, a suscribir convenios diversos tendientes a mejorar la formación de recursos humanos y obtener ventajas para los participantes, en fin, a promover la internacionalización de sus estudios como sinónimo de modernización.

La estructura de las licenciaturas en Contaduria Pública, debe vincularse a la internacionalización de la economía y a las necesidades locales. Nuestra intención es ofrecer un plan de estudios latinoamericano con elementos de formación básicos aplicable a todas nuestras instituciones. Estamos seguros que compartimos una base teórica común, que aplicamos enfoques metodológicos semejantes y que tenemos los mismos retos éticos en cada una de las empresas y organizaciones en que laboramos.

En estas condiciones, la formación del Contador Püblico reviste singular importancia, dadas las condiciones actuales de las economias en los países de América Latina. sujetas cada vez más a los vaivenes del neoliberalismo que propicia la concentración de la riqueza, la corrupciơn y la ineficiencia.

Es nuestra obligación reflexionar sobre el papel de este profesional y replantear sus responsabilidades y las modalidades en su formación en los aspectos siguientes:

a) Fomentar la eficiencia de nuestras empresas, públicas o privadas

b) Participar en la generación de empleos

c) Promover el desarrollo sustentable

d) Abatir la corrupción mediante la implantación de controles eficientes y la realización de auditorias veraces e independientes

e) Contribuir a la recaudación de las contribuciones para el gasto público

f) Permitir la participación en la generación de la riqueza y su adecuada distribución. 


\section{OBJETIVO}

Integrar, por medio de una consulta regional, una propuesta minima para orientar los planes y programas de la licenciatura en Contaduria Pública que ofrecen las Instituciones de Educación Superior en América Latina.

\section{MECANISMO DE CONSULTA} a tratar:

Se podrian considerar dos opciones base, teniendo previamente el acuerdo sobre los temas

1. Coloquios generales en cada uno de los paises participantes.

Logros: Se tendria una mayor cantidad de comentarios y propuestas, además de permitir el conocimiento de todo el proyecto por cada pais participante.

2. Distribución de los temas entre regiones y efectuar coloquios regionales, de acuerdo a la estructura ya aprobada por ALAFEC.

Logros: Menos tiempo para concretar las propuestas y menores costos.

En cualquiera de los casos la consulta se efectuaria por medio de COLOgUIOS, en las siguientes etapas:

Primera Etapa: Diagnóstico de la situación actual en las instituciones de educación superior participantes, previo acuerdo de la información relevante necesaria para el estudio.

Objetivo: Obtener un panorama general de la situación actual de los planes y programas de estudio de la Contaduria Pública en América Latina, los principales modelos de organización curricular, su misión y bases psicopedagógicas, los perfiles de ingreso y egreso, los conocimientos básicos sustantivos, las lineas de investigación y los mecanismos de evaluación y acreditación. Este estudio nos mostrará semejanzas y diferencias en los enfoques de la enseñanza de la contaduria.

Metodologia: Elaboración y aplicación de un cuestionario para solicitar la información acordada. Se anexa propuesta de cuestionario.

Responsables: Representantes de cada pais participante en colaboración con la Coordinación Regional respectiva.

Segunda Etapa: Estudio de los temas que formarán la ponencia central.

Objetivo: Ofrecer un foro de participación y discusión a los académicos de la Contaduria Pública en América Latina para diseñar una propuesta académica orientadora de los planes y programas de estudio. Los resultados de estas reuniones serán presentados en la Asamblea de ALAFEC en la Ciudad de México, en septiembre de 2003.

Metodologia: Celebración de 4 coloquios para discutir los siguientes temas, en el entendido de que se solicitará previamente a los académicos participantes un documento con el desarrollo de los temas seleccionados. La asistencia al coloquio dependerá de la aprobación de este documento.

\section{Primer Coloquio:}

- Definición de las áreas básicas de conocimiento en la formación del Contador Público y la razón de su particularización. Puede ser útil en este aspecto tomar en consideración y discutir sobre las competencias, habilidades y capacidades técnicas del profesional, de la contaduria pública. Establecer de manera precisa cuáles labores y actividades se deben considerar de la competencia en el ejercicio de la profesión.

- Definición de las áreas de conocimiento complementarias en la formación del Contador Público. Hay que identificar los conocimientos minimos requeridos en las áreas de economia, mercadotecnia, administración, de preferencia, asi como los métodos e instrumentos de trabajo de las ciencias sociales.

- Los requisitos minimos en los planes y programas de estudio de la licenciatura en contaduria.

- Analizar las modalidades más convenientes para los programas, considerando de manera muy importante la posibilidad de convalidar los titulos emitidos en diversos paises y las opciones en la movilidad profesional.

Segundo Coloquio:

- El Contador Público, la administración pública, la empresa y sus paradigmas.

Sobre este particular se sugiere la consideración de los nuevos paradigmas vigentes, a 
partir de los acuerdos internacionales de comercio, en adición a las normas regionales y principios generalmente aceptados.

- La función social de la empresa.

Estudiar al más alto grado de desagregación los aspectos relativos a la función social de la empresa, sus componentes fundamentales y lo referente a la responsabilidad social de la entidad empresarial, haciendo énfasis en las caracteristicas que muestran empresas de sectores especificos en la región, asociadas a las políticas públicas y a sectores de alta sensibilidad, así como a programas especiales de combate a la pobreza auspiciados por gobiernos locales, nacionales $\mathbf{u}$ organismos internacionales que pueden ser modelos a considerar en el tratamiento académico de la problemática vinculada con el ejercicio profesional de la contaduria pública.

- La Contaduria Pública Latinoamericana ante el contexto internacional.

Se propone la realización de un amplio diagnóstico que permita a la asociación abordar el desarrollo de una agenda regional de trabajo para estandarizar conceptos, normas y principios de trabajo y de competencia laboral.

Tercer Coloquio:

- La formación ética para el desempeño profesional del Contador Público en América Latina.

- La responsabilidad social del Contador Público.

Analizar, discutir y aprobar la carta ética del Contador Público para Latinoamérica, asi como la articulación y adopción de un conjunto de criterios para su desempeño, que permitan establecer el perfil de la responsabilidad social del contador público.

- El ejercicio profesional del Contador Püblico ante la Corrupción. generación de confianza. Se propone un examen detallado de los procedimientos de rigor en el levantamiento de auditorias a instituciones y procesos, asi como la observación de normas y técnicas científicas que guian los mismos.

Cuarto Coloquio:

- La investigación científica como actividad del Contador Püblico.

Se reconoce el interés de auspiciar un amplio programa de investigación científica e coordinación con otras, entidades regionales o nacionales, vinculadas al quehacer cotidiano de la contaduria pública. Reflexionar sobre el sentido y significado de la Investigación para nuestra disciplina.

- Los Postgrados en las áreas de la Contaduria. (Multidisciplinario)

$\mathrm{Si}$ queremos mejorar los procesos de formación de contadores en América Latina es necesario ponerle más atención al Postgrado, máximo nivel educativo que ofrecemos y que por sus caracteristicas y objetivos particulares puede influir positivamente en la enseñanza y el desarrollo de la contaduria. En la actualidad, el grado de especialización en estas áreas es minimo y desarticulado, incluso aun en cada país. Se estima de gran interés la incorporación de programas regionales de Postgrado que contribuyan a fortalecer la calidad en el ejercicio de la profesión.

Responsables: De acuerdo a la opción seleccionada, en el primer caso el responsable será el representante de cada país; en el segundo caso, será el Coordinador Regional, apoyado por los representantes de los paises que formen esa región.

\section{REgLAMENTO GENERAL PARA los COloguios, ALAFEC}

Para obtener la opinión y justificación de los profesores especialistas de las instituciones afiladas a la ALAFEC y de los organismos profesionales de Latinoamérica se acordó realizar coloquios para discutir la formación del Contador Publico en los temas siguientes:

- Las áreas de conocimientos básicos y complementarios de la licenciatura.

- La empresa y sus paradigmas.

- La función social de la empresa.

- La Contaduria Pública Latinoamericana ante la Globalización.

- La ética y la responsabilidad social del Contador Público.

- El Contador Público y la auditoria de instituciones y procesos.

- La investigación.

- El Postgrado en áreas de la Contaduria Pública.

El reglamento tiene XV capítulos y transitorios fijándose como fecha limite para el desarrollo de los coloquios el mes de junio del año 2003. 


\section{CREACIÓN DE LA RED INTERNACIONAL DE INVESTIGADORES EN CONTADURIA DE LA ALAFEC (RIIALAFEC)}

El proyecto presentado por la Comisión respectiva es el siguiente:

\section{ANTECEDENTES}

Consciente de que es impostergable que se dé un impulso vigoroso a la investigación en contaduria en las universidades latinoamericanas con el fin de generar conocimiento en lugar de sólo transmitirlo acríticamente, la ALAFEC ha decidido crear su Red Internacional de Investigadores en Contaduria (RIIALAFEC). Dicha Red se funda en la premisa de que el avance del conocimiento en cualquier disciplina históricamente se ha dado y se seguirá dando sólo gracias al diálogo entre los investigadores de la misma, el cual comprende no sólo el que establecen oralmente con sus colegas o pares, sino también y principalmente el que entablan a través de la palabra escrita con pensadores presentes, pasados e incluso futuros.

La necesidad de dicho proceso, que implica el conocimiento y análisis del pensamiento de otros, queda claramente ejemplificada en el multicitado pensamiento de Newton: «Si he visto más allá, es por haberme colocado en los hombros de gigantes".

Hasta hace poco tiempo, unas dos décadas, dicho diálogo se realizaba cotidianamente a través de lo dicho por los investigadores en libros, revistas especializadas y, en algunos casos, en contactos personales; ocasionalmente se llevaba a cabo, además, por medio de eventos académicos diversos (congresos, simposios, conferencias, etc). Todos estos medios que hoy en dia siguen y continuarán siendo por largo tiempo empleados para el desarrollo del conocimiento científico y técnico, han recibido un impulso importante de los nuevos medios electrónicos que facilitan y aceleran el intercambio de pensamientos entre los investigadores. Estos medios electrónicos sin duda habrán de ayudarnos también en la Red Internacional que estamos creando, pero de ninguna manera substituyendo a los medios tradicionales, sino sölo apoyándolos como vehículos de comunicación.

\section{OBJETIVOS}

Las redes de investigadores coadyuvan a la investigación facilitando el intercambio de ideas entre aquéllos y la realización de proyectos de investigación conjuntos. En el caso concreto de la ALAFEC, su Red Internacional de Investigadores en Contaduria busca alcanzar los siguientes objetivos:

Impulsar la investigación tanto básica como aplicada en contaduria a través de:

- Facilitar y multiplicar el intercambio académico de pensamientos y hallazgos entre los investigadores latinoamericanos, principalmente por medio de artículos, ensayos y sugerencias bibliográficas, así como de criticas y consultas mutuas.

- Promover la realización de proyectos de investigación conjuntos.

- Promover la participación de los investigadores latinoamericanos en eventos académicos de los diferentes países, así como el intercambio de investigadores, estudios y materiales de investigación.

\section{ACCIONES INICIALES PROPUESTAS}

\section{Elaboración del Directorio Latinoamericano de Investigadores en Contaduria}

Para el establecimiento de la red es fundamental la elaboración de un Directorio Latinoamericano de Investigadores en Contaduria que se deberá ir alimentando de directorios nacionales que ya existan o que elaboremos en cada uno de nuestros países. De hecho, dicho Directorio será el instrumento central o corazón de la Red.

Para la elaboración del Directorio proponemos que se lance una convocatoria general para Latinoamérica y que en cada país se lance a su vez una convocatoria nacional para la conformación de los directorios correspondientes.

De hecho, en México, a través tanto de la Facultad de Contaduria y Administración de la UNAM, como de la Asociación Nacional de Facultades y Escuelas de Contaduria y Administración (ANFECA), hemos realizado ya algunos avances para la creación del Directorio Nacional de Investigadores en Contaduria y Administración.

Para que sean instrumentos prácticos y de relativamente fácil actualización, tanto el directorio latinoamericano como los directorios nacionales deberán contener poca información pero muy pertinente. Si ésta es complicada el directorio y la red se volverán pronto obsoletos. Se estima que la información necesaria acerca de cada investigador es la siguiente: 
- Nombre y demás datos de identificación del investigador (domicilio, dirección de correo electrónico, teléfono[sl).

- Institución en que labora

- Área(s) de investigación.

- Línea(s) de investigación que trabaja.

- Títulos de los trabajos que ha realizado en la misma línea de, investigación y referencias de su publicación, en su caso.

- Proyecto de investigación actual y proyecto(s) futuro(s)

Con el objeto de uniformar los criterios respecto a lo que deberá entenderse por área y línea de investigación, lo cual es de suma importancia, al lanzar las convocatorias se incluirán las siguientes definiciones:

Ảrea de investigación. Rama o Campo de conocimiento de la contaduria sobre el cual se pretende realizar investigación, y que comprende una o varias líneas de investigación afines. Por ejemplo, las áreas de finanzas, auditoria, costos y contribuciones.

Linea de investigación. Tema o problemática especifica de investigación de un área, dentro del cual se pueden inscribir una infinidad de proyectos de investigación individuales o colectivos. Su fin primordial es el de señalar y delimitar a muy grandes rasgos, mediante un simple titulo, un tema o problemática de interês de un investigador y/o un centro de investigación.

Por ejemplo: métodos de valuación de empresas, como linea del área finanzas corporativas; principios de contabilidad nacionales e internacionales, como linea del área contabilidad; costos $\mathrm{ABC}$ como línea del área de costos; teoria del portafolios de inversión, como línea del área de teoría financiera.

\section{Creación de un órgano coordinador de la Red}

Se propone la creación de una Coordinación de la Red Internacional de Investigadores en Contaduria, ALAFEC, con las siguientes funciones principales:

Funciones transitorias

- Continuación de la elaboración del presente proyecto y coordinación de las actividades que deban efectuarse para convertirlo en realidad una vez que sea aprobado.

- Elaboración de una propuesta de página de Internet de la Red y establecimiento de una dirección de correo electrónico de la misma.

- Apertura de la página de Internet de la Red una vez que haya sido aprobada.

- Elaboración de la propuesta de convocatoria para conformar el Directorio Latinoamericano de Investigadores en Contaduria.

- Publicación de la convocatoria para la conformación del Directorio citado en diversos medios. incluyendo la propia página de Internet de la Red.

- Depuración y ordenación de la información obtenida en respuesta a la convocatoria lanzada y publicación de la misma como Directorio Latinoamericano de Investigadores en Contaduria.

Funciones permanentes

Coordinación de todas las actividades tendientes a la operación de la Red, asi como a su actualización y mejora

Promoción para lograr que la Red se amplie mediante la inscripción de nuevos investigadores.

AFILIACIÓN DE INSTITUCIONES A ALAFEC

Anexo 04

\section{PROYECTO PARA EL POSICIONAMIENTO, AFILIACIÓN Y CONSOLIDACIÓN DE LA ALAFEC}

El Consejo Ejecutivo de la ALAFEC debe iniciar de manera decidida una campaña de posicionamiento y afiliación de instituciones que permita la consolidación de nuestra Asociación.

\section{OBJETIVOS:}

El proyecto para el posicionamiento, la afiliación y consolidación que debe emprender el Consejo Ejecutivo de la ALAFEC, debe tener tres objetivos básicos: 
1. Posicionar la imagen de ALAFEC en Latinoamérica.

2. Afiliar al mayor número posible de Facultades y Escuelas de Contaduria en Latinoamérica.

3. Lograr que la membresía afiliada asista a la próxima Asamblea General de la ALAFEC. El éxito de la próxima Asamblea permitirá consolidar a nuestra Asociación.

Se explican a continuación:

1. Posicionar la imagen de ALAFEC en Latinoamérica

La imagen que debemos proyectar es la de una Asociación académicamente sólida que promueva, por medio del conocimiento y colaboración mutua, la vinculación de las Facultades y Escuelas de Contaduria en América Latina.

Esta imagen se ve respaldada, tanto por el prestigio de las instituciones que integran el Consejo Ejecutivo, como por el correcto cumplimiento de los proyectos y objetivos señalados en el Plan de Trabajo 2002-2003.

ESTRATEGIA BASE:

- Difundir en toda América Latina la Gaceta de ALAFEC, como órgano oficial de comunicación cuyo primer nümero explica el ser y quehacer de nuestra Asociación.

2. Afiliar al mayor número posible de Facultades y Escuelas de Contaduría en Latinoamérica Las instituciones que soliciten su afiliación deben ajustarse a las disposiciones contenidas en los Estatutos de la Asociación y al Procedimiento de Afiliación correspondiente.

\section{ESTRATEGLAS:}

Para la afiliación de instituciones se propone seguir las siguientes estrategias.

a) Estrategia Nacional.

Los Representantes Nacionales serán los responsables de realizar una campaña de afiliación en cada uno de sus paises.

Se considera conveniente establecer objetivos cualitativos y cuantitativos. De manera cualitativa el objetivo será conservar el prestigio de nuestra membresía, por lo que se permitirá la afiliación únicamente a aquellas instituciones que cumplan con los requisitos y el procedimiento establecido para tal efecto.

Por otra parte, de manera cuantitativa, se propone que, a partir de esta fecha y hasta la próxima reunión de este Consejo, cada Representante Nacional afilie a un minimo de 5 instituciones.

b) Estrategia Regional.

Los Vicepresidentes Regionales se encargarán de designar, en los países donde haga falta, al Representante Nacional correspondiente. Dicho Representante Nacional adoptará el compromiso de llevar a cabo la campaña de afiliación en su país bajo la asesoria del Vicepresidente que le corresponda.

c) Estrategia Central.

La Presidencia de la Asociación, a través de la Secretaria Ejecutiva y Coordinación de Relaciones Internacionales, impulsará la campaña de afiliación por medio de una estrategia que contempla hacer llegar una exhortación a los Rectores de las Universidades que formen parte de la Unión de Universidades de América Latina (UDUAL) con el fin de que apoyen e impulsen a los Directores o Decanos a formar parte de esta Asociación.

La Secretaria General, se responsabilizará de hacer llegar, de manera ágil y oportuna, el Dictamen Definitivo de Afiliación a las instituciones solicitantes. Así mismo, y de manera conjunta con la Coordinación de Relaciones Internacionales, se asignarán registros oficiales, elaborándose una base de datos con las nuevas instituciones afiliadas.

3. Lograr que la membresía afiliada asista a la próxima Asamblea General de la ALAFEC. EI éxito de la próxima Asamblea permitirá consolidar nuestra Asociación

La Coordinación de Relaciones Internacionales se responsabilizará de mantener informada a la membresía de la Asociación sobre los aspectos referentes a la próxima Asamblea General de la ALAFEC. 